\title{
Afterword: COVID-19 and Senior Care and Services
}

\section{INTRODUCTION}

The COVID-19 global pandemic has had a major impact on the world, and the United States has been struck particularly hard with the first wave of reports starting in February and community spread continuing well into the summer and early fall. Senior living has been hit by a perfect storm with the virus being both contagious and spread by asymptomatic and symptomatic staff or visitors to these congregate settings serving a frail and vulnerable population.

As would be expected with the introduction of a new and fast communicable virus, many of the cases and deaths are occurring in skilled nursing facilities, with significant outbreaks and deaths also being reported in both assisted living facilities and senior housing. The special needs of residents also present significant challenges in managing this population's complexities of care and service needs. Compounding these factors has been the substantial shortage of personal protective equipment (PPE) made available for senior care communities. The focus on hospitals has certainly been highlighted by federal and state leadership priorities, yet the PPE needs of senior care providers have been underemphasized in the public dialogue. The elders that we serve deserve the focus and attention that this pandemic demands. Senior care communities already had faced staffing challenges prior to the pandemic, and this disease has only amplified the pressing needs in these facilities. Staff need to feel respected, safe, and valued by both their own organizations and society as a whole. We have let them down. Administrative leaders have worked tirelessly to support these often-unsung heroes. Misconceptions compounded by poor communication coming from a variety of sources, such as governmental leaders and news outlets and social media, have further added fuel to a fire that has required caring, proactive messages of hope and support to staff. Further, there is mixed evidence about whether the quality of care centers is correlated with infiltration or spread of COVID-19 (Abrams, Loomer, Gandhi, \& Grabowski, 2020; Li, Temkin-Greener, Gao, \& Cai, 2020). The appropriate role of the government should be one of assistance and support, not one of overly aggressive scrutiny and levying of fines (Centers for Medicare and Medicaid Services [CMS], 2020a). There has been some evidence that CMS and state health departments have been attempting to move in this direction (CMS, 2020b).

The senior living provider community must reconsider inconsistent admission and discharge policies and approaches that have not always served the best interests of their residents and patients. Treatment protocols have been advanced with 
only modest scientific support, which has further added to a confusing environment. There have also been some providers that have not advanced or maintained the proper infection control processes nor have monitored adherence to the proper use of PPE. Overall, there are many who should be held accountable for not providing adequate support for direct care staff who have served-or are now servingpatients on the frontlines of the fight.

There are some things we learned quickly that helped the senior care community weather the storm. First and foremost, access to adequate PPE is a necessary factor for success. Second, the importance of having a strong infection control training program for all staff in place cannot be underestimated. One of the key related success factors has been to have adequate testing available for both consumers and staff. Another necessary condition has been a strong leadership presence, including the administrator, medical director, and clinical leadership. There has also been some success with using COVID-19 designated facilities across the country, especially among multisite organizations. For many providers, using isolation strategies has been an internal approach that has yielded positive results in controlling the spread of this virus. An example of a related practice effort has been to make sure that advance directives are in place and updated using a frailty assessment, especially with patients who are potentially at higher risk. (Buslovich, Nazir, \& Wasserman, 2020).

There are several lessons that have been relearned by organizations. These areas have been amplified during this pandemic and, although covered in the text, deserve additional emphasis related to the situation the senior living field finds itself in for the foreseeable future.

- Infection control and prevention is a critical practice that has been highlighted and will need to be solidly in place going forward. Protocols as simple as promoting good handwashing techniques and following appropriate precautions are essential going forward. These approaches also send a positive signal to your staff that you are committed to taking care of them as caregivers.

- One of the bigger lessons has been the practice of effective and efficient operational communications. The variety of stakeholders and the limitations on in-person interaction because of the pandemic have required different communication approaches. Due to the ever-changing nature of this situation, the senior living field finds itself needing to provide regular, weekly, or even daily informational messages to residents, tenants, clients, and staff to ease apprehensions and provide clarity for the road ahead. The importance of communicating with families of all of these groups has also been amplified, answering questions and boosting confidence that the leadership is doing everything possible to ensure a safe environment and remain as attentive and accessible as possible. Leaders must also communicate appropriately to their governing boards and corporate ownership-garnering their support during this unprecedented event yet avoiding the unintentional inclination to overcommunicate and for them to get immersed into the tall weeds. Leadership needs to balance what constitutes enough and too much information. When communicating to the broader community, the specific content of the information being communicated should determine the approach. Pausing to evaluate what you are trying to accomplish or convey is a first step with all of these messages. 
- Paying attention to the broader wellness factors of your audiences has taken on a whole new meaning during this pandemic. The most obvious is their physical well-being, which has had to be front and center, especially for residents and clients, along with employees and volunteers. The mental and emotional toll this has taken on everyone requires both thought and action, in the form of resources made available to help individuals cope and survive this challenging time. The changes to the conventional religious gathering and methods for providing spiritual support have added challenges for many already facing hardships. Lastly, the economic struggles go well beyond just one's own organization, as just about every family circle has felt some of the financial stresses of this period.

Successful leaders must be tuned into many of these reemphasized facets of organizational life when running their healthcare businesses. It has taken extra energy and insight to navigate these uncharted waters.

Lessons revealed during this pandemic have touched a wide array of issues, and many have had a significant impact on the senior care services field. They include the broad categories of infection control, coordination, operations, staffing, and holistic wellness.

\section{INFECTION CONTROL AND PREVENTION}

Beyond the health precautions required of everyone, the importance of paying attention to community spread factors is critical. The world also understands better the many ways in which we are interdependent when it comes to travel and shipping. Social distancing, often now called "physical distancing," is a new construct that we have all gotten to know. This has had a major impact on senior care services, especially concerning visitors and memory care venues. Quarantining for 10 to 14 days after coming into contact with an individual with COVID-19 or even visiting a community that has confirmed cases is a new expectation for any person entering the building who does not reside there. Another new concept has been the policies required to limit group assembly, which has been viewed in some settings as one that has competing rights: one's civil liberties versus another's right to remain healthy. For purposes of senior care, the public health field has weighed in heavily with the limitations of these gatherings or visits to help protect the vulnerable population of senior care communities. This has been a challenge for both providers and families. Lastly, we have learned that senior living belongs on the list of businesses considered "essential" during a pandemic.

There have also been clinical protocols that have been important to practice after using them only infrequently and to learn because they were unfamiliar in senior living settings. Two of the most obvious examples have been proactive diagnostic testing and widespread use of PPE (e.g., particularly masks and eyewear). Testing for the presence of the virus provides an opportunity for early detection, but it is only a snapshot in time. Testing survivors for the antibody gives some assurances that they are unlikely to remain contagious - even if still symptomatic-but we do not have enough evidence accumulated yet about how long they will remain so. How do you ensure that you have an adequate supply? How do you administer or encourage tests? What do you do when a staff member or resident (or his/her guardian) refuses to test? How do you use the information provided by the results? 
There is also a matter of priority-setting for the use of tests, depending on the supply. The "when" and "frequency" of testing are also important considerations. An organization must have a contingency plan for responding if it receives positive results. Federal and state agencies issued inconsistent-sometimes conflictingguidance about whether to rely on a symptom-based or test-based strategy for resident admissions, discharge, or transfer, as well as for personnel returning to work.

Given COVID-19's multiple avenues of transmission, PPE has been crucial (Centers for Disease Control and Prevention, 2020). The availability and distribution of these supplies have been an issue, and the supply chain has been tested on multiple levels. A few of the issues have been:

- appropriate supply levels on hand,

- setting priorities and tiers for distribution,

- the competition among states for access to the federal stockpile, and

- fluctuations in actual market prices.

At the provider level, inventory management has been tested, including how to:

- initiate real-time inventory tracking,

- calculate the right "burn-rate," and

- manage all the logistics so customers and staff are safe and have the appropriate PPE.

\section{COORDINATION}

This is a tale of success (and the lack thereof) with both the public and private sectors. Starting with government agencies, there have been new kinds of challenges for the coordination of support among federal, state, and local levels. Emergency manufacturing and distribution of desperately needed ventilators and PPE, as well as providing some shared staffing support, were accomplished with remarkable speed, but with significant disparities among regions and levels of care. Members of the healthcare provider continuum have also been tasked with working better within their ranks to communicate and manage COVID-19 patients. The role of suppliers that provide vital goods, from pharmaceuticals and medical equipment to food and cleaning products, has brought tremendous value to organizations. Even the ancillary services, such as utilities, trash collection, communications, education, and electronic health technology, have all adjusted to support people and organizations. The communication and coordination of all these resources and services have been magnified. The Trump Administration even directed the CMS to create an Independent Coronavirus Commission on Safety and Quality in Nursing Homes (CMS, 2020c).

\section{OPERATIONAL COMMUNICATION}

Communication has been front and center for senior care leaders with this pandemic, with lots of stakeholders. Staff need to be assured that you are doing everything you can to keep them safe. They also need to know that you appreciate their efforts. Residents, tenants, clients, and their families need many of the same messages of 
assurance of their safety. They also need to know that you care and understand their anxieties and fears. The more formal communications with agencies and the public media are largely focused on specific requests and reactions. One of the frustrations is that the general media continues to play the blame card more often than not, and of course negative news attracts more attention (in most cases). The role of an effective leader is to wade through some of this news and create some calm by not yelling "Fire" in a crowded theater. It is also clear that greater transparency helps to squelch the fear of the unknown that was very present in the early stages of the pandemic. Lastly, being proactive with communication and transparent with all efforts has been critically important.

\section{STAFFING}

The real heroes of this pandemic have been the frontline staff. They have accepted the risk and cared for the elders that deserve our very best. A number of proactive management decisions have helped, including implementation of dedicated assignments, tracking of multisite workers (within the same organization or among multiple employers), provider-issued transportation (rather than reliance on public transportation or rideshare), and even creation of temporary staff housing to protect staff families from COVID-19 transmission. The communities we serve have also responded with tremendous support of our staff. There have been many stories of communities and families reaching out to help support staff with meals and childcare assistance.

This environment has also required the mobilization of human resources from a variety of sources to help fill the staffing needs caused by vacant positions or staff illness. The National Guard, unemployment insurance enrollees, furloughed health professionals (especially from acute care), and college students have all represented emergency sources of labor. One of the by-products of using temporary staff has been modest relaxation of some licensure standards, which has relied on the flexibility and sound judgment of state licensing boards and the related health professions. As an example, there has temporarily been a reduction of the training requirements for certified nursing assistants. This has long-term implications, depending on when and how the normal standards are reinstated, for the public protection afforded by licensure concerning qualifications, credentials, and regulatory oversight.

The governing boards and owners of these care and service organizations are also facing a new fiscal climate, and their budgets have had to both evolve and be refined. This has also required a new engagement with public officials to ensure the most appropriate support during such an unanticipated world event. The guidance and support for executive leadership have had to be at the forefront of these boards and corporate owners as they weather the storm of COVID-19.

\section{HOLISTIC WELLNESS}

A new essential perspective required of leadership - to take care of the caregivers, care recipients, and families of both-is a broader view of the individual and the organization's role in their lives. This includes considering their physical, mental, emotional, spiritual, and economic health. In some cases, it means considering how 
to support them to meet their basic security needs. What happens to the lifelong nursing assistant who exhausts all sources of supplemental income, such as paidtime-off and unemployment benefits, because he or she contracts COVID-19 while working for a senior living organization? These are difficult choices for an organization, but ones that can readily emerge. This has been a difficult time for our country, with many false choices, such as pitting the reopening of the economy against people surviving and living. Our field has risen to the challenge and has nobly responded with compassion and common sense.

\section{LESSONS TO APPLY GOING FORWARD}

As a country, we have certainly been reminded of the need to invest in our public health infrastructure. There will be much work ahead to revise public health protocols, policies, and procedures. We must blend these efforts with the healthcare system's emergency disaster planning infrastructure. We must also invest in preparation for similar events in the future. This should include contingency planning, supply chain management and inventory control for supplies and equipment, a training and distribution strategy for people, supplies and equipment, and a better, more seamless and technology-assisted approach for contact tracing. People are also taking a step back to reconsider our reliance on foreign resources and reevaluating the necessary funding level needed for truly fostering public health.

Regarding our senior living field, we have made great strides with a number of new or renewed practice efforts. The use of designated COVID-19 facilities or units, often described as Centers of Excellence, has been a shared healthcare system accomplishment. The use of remote patient monitoring, novel data-driven testing, and the development of frailty-driven care pathways for COVID-19 patients are all examples of strides forward. There is a renewed emphasis on training for infection control and a broader perspective on disaster preparedness, as various systems have embraced the value of proactively addressing these new threats for the benefit of our customers, staff, and organizations.

Although it may be a reach to identify any positives while facing this pandemic, several good things have emerged as we reexamine how we do things. The following list captures a few of these approaches or practices that we can possibly reimagine in the future.

- The need for reconsidering congregate living may leave us in place where semi-private rooms in skilled nursing facilities may not be an acceptable practice or policy. This change will have both quality and price considerations, but the time for the discussion is now.

- Relationships among the acute care system, senior care providers, and public health agencies will need to continue to develop and evolve, especially after great progress has been made.

- Communication modes for all stakeholders will get better. The use of telemedicine will not only be maintained but will be expected to improve as we press forward. Families will communicate with loved ones more frequently, with access to and use of technology becoming even more prevalent.

- A new, broader look at crisis-related staff assistance and trauma-informed supports provided by both employers and the broader society will emerge. 
The value of senior care as an "essential" service will continue to be more widely understood, although there is no question that we need to continue to press forward with building the respect this field deserves.

There will be many more new and reimagined opportunities that present themselves in the next few years, and there is still a lot of hard work ahead of us.

In closing, we can only scratch the surface in our expression of all the amazing efforts and stories of this often-unsung field of senior care and services during the pandemic. We appreciate the many people across the country doing this work every day. We are hopeful that America will make the choice that the elders of today and tomorrow-and all of their caregivers across the country-deserve the very best support. This is a time in our country where actions speak louder than words.

\section{REFERENCES}

Abrams, H., Loomer, L., Gandhi, A., \& Grabowski, D. (2020). Characteristics of U.S. nursing homes with COVID -19 cases. Journal of the American Geriatrics Society, 68(8). doi:10 $.1111 /$ jgs.16661

Buslovich, S., Nazir, A., \& Wasserman, M. (2020, June 30). COVID-19 learnings and strategies from medical directors on the front lines. Pathway Health and Patient Patterns webinar.

Centers for Disease Control and Prevention. (2020). How COVID-19 spreads. Atlanta, GA: Author. [Google Scholar]

Centers for Medicare and Medicaid Services. (2020a). Trump Administration unveils enhanced enforcement actions based on nursing home COVID-19 data and inspection results. https: / / www.cms.gov/newsroom/press-releases/trump-administration-unveils-enhanced -enforcement-actions-based-nursing-home-covid-19-data-and

Centers for Medicare and Medicaid Services. (2020b). Trump Administration announces new resources to protect nursing home residents against COVID-19. https: / / www.cms.gov/ newsroom / press-releases / trump-administration-announces-new-resources-protect -nursing-home-residents-against-covid-19

Centers for Medicare and Medicaid Services. (2020c). CMS announces membership of Independent Coronavirus Commission on Safety and Quality in Nursing Homes. https: / / www.cms.gov / newsroom/press-releases / cms-announces-membership-independent-coronavirus -commission-safety-and-quality-nursing-homes

Li, Y., Temkin-Greener, H., Gao, S., \& Cai, S. (2020). COVID-19 infections and deaths among Connecticut nursing home residents: Facility correlates. Journal of the American Geriatrics Society. doi:10.1111/jgs.16689

\section{ADDITIONAL RESOURCES}

There is a vast array of association-related resources available for providers.

Centers for Disease Control and Prevention: Coronavirus Disease 2019 (COVID-19): Retirement Communities: https: / / www.cdc.gov / coronavirus / 2019-ncov / community / retirement/ index.html

Centers for Medicare \& Medicaid Services: Toolkit on State Actions to Mitigate COVID-19 Prevalence in Nursing Homes: https: / / www.cms.gov/ files / document/ covid-toolkit -states-mitigate-covid-19-nursing-homes.pdf

AARP: Family Caregiving: Protect Nursing Home Residents: https: / www.aarp.org/caregiving/ nursing-homes / 